\title{
Reverse-engineered silk hydrogels for cell and drug delivery
}

\author{
F. Philipp Seib ${ }^{1,2}$ \\ ${ }^{1}$ Strathclyde Institute of Pharmacy and Biomedical Sciences, University of Strathclyde, \\ 161 Cathedral Street, Glasgow, G4 0RE, UK \\ ${ }^{2}$ Leibniz Institute of Polymer Research Dresden, Max Bergmann Center of Biomaterials \\ Dresden, Hohe Strasse 6, 01069 Dresden, Germany
}

F. Philipp Seib; Tel: +44 141-548-2510; Fax: +44 141-552-2562; emails:

philipp.seib@strath.ac.uk or philipp.seib@SeibLab.com

\section{Acknowledgement}

This research was supported in part by a Marie Curie FP7 Career Integration Grant 334134 within the $7^{\text {th }}$ European Union Framework Program and an EPSRC First Grant EP/N03127X/1. 


\begin{abstract}
Silk is an important biopolymer for (bio)medical applications because of its unique and highly versatile structure and its robust clinical track record in human medicine. Silk can be processed into many material formats, including physically and chemically crosslinked hydrogels that have almost limitless applications ranging from tissue engineering to biomedical imaging and sensing. This concise review provides a detailed background of silk hydrogels, including silk structure-function relationships, biocompatibility and biodegradation, and it explores recent developments in silk hydrogel utilization, with specific reference to drug and cell delivery. We address common pitfalls and misconceptions while identifying emerging opportunities, including 3D printing.
\end{abstract}

Key words: Silk fibroin, hydrogel, self-assembly, stem cells, B. mori, tissue engineering 


\section{Introduction}

Silk has a unique and highly versatile biopolymer structure that makes it ideal for biomedical applications. It is particularly useful for cell and drug delivery, because it can be processed into many material formats, including fibres, fabrics, films, scaffolds, hydrogels and micro- and nanoparticles [1]. Furthermore, this high-performance biomaterial is already FDA/EMA/MRHA clinically approved (for load bearing applications), and has renowned biocompatibility, tuneable biodegradation (minutes to 12 months and more) and low immunogenicity [2]. Its unique physical properties (e.g. toughness) have long supported its use as a suture material (e.g. Ethicon, Inc.) and more recently as a surgical mesh for load-bearing applications (Allergan, Inc.) [3]. In China, the Sidaiyi ${ }^{\circledR}$ wound dressing (i.e. a silk sponge attached to a silicon film) is licensed for human use in donor site partial- and full-thickness wounds, donor site wounds and burns. Further expansion of silk use is evident in ongoing clinical proof-of-concept studies available in the public domain: (i) silk-based meshes for breast reconstructive surgery [4] and (ii) silk films as a wound dressing [5]. Commencement of a Phase I clinical study using silk coatings of silicone breast implants for improved host-tissue response (BioShield-S1, AMSilk GmbH Germany) is expected shortly.

Overall, silk has a robust safety record, making it a highly attractive material for state-of-the-art medical applications, including stimulus-responsive nanomedicines, payload stabilisation (drugs, proteins and diagnostics; developed by Vaxess Inc., MA, USA), medical sensors, hydrogels for drug and cell delivery, tissue engineering and in 
vitro disease modelling. This review does not attempt to provide a comprehensive review of all silk hydrogels proposed for drug and cell delivery/tissue engineering nor does it assess in detail the manufacturing techniques used (these have been reviewed elsewhere; e.g. [6]). Instead, the aim of this review is to deliver a critical assessment of the current opportunities and challenges of silk hydrogels and to provide a wider context for their use.

\section{Silks}

In this review, the term silk refers to protein-based fibre-forming materials spun by living organisms. Spiders and silkworms are the most prominent organisms associated with silk production, although silks are made by many other organisms $[7,8]$. This review will critically examine the literature related to Bombyx mori silk.

B. mori is the domesticated silkworm that is exploited for commercial sericulture (i.e. silk

farming). For the purpose of this review, we will focus on silkworm silk (Fig. 1) because this is the most common silk used in ongoing biomedical research due to its ready availability and it is currently in clinical use in humans for load-bearing applications [1].

\section{Bombyx mori silk}

The exact molecular size of silk varies among different $B$. mori strains due to variations in the lengths of the crystalline coding domains in the silk heavy chain [10]; therefore, the reported variations in molecular weight are potentially due to biological variability and/or differences in experimental approaches. The B. mori silk consists of a heavy chain (approximately $391 \mathrm{kDa}$ ) [11, 12] and a light chain (approximately $26 \mathrm{kDa}$ ) [13], which 
are held together by a single disulphide bond at the C-terminus [14] (Fig. 1b). The mechanical properties of silk arise from the unique amphiphilic protein structure of its heavy chain, which includes 11 short hydrophilic regions that are typically 31 amino acids long, together with hydrophilic $\mathrm{C}$ - and N-terminal capping sequences consisting of completely non-repeating amino acid residues (Fig. 1). The 11 hydrophilic regions are interspaced within the heavy chain, which consists of 12 hydrophobic blocks that account for $94 \%$ of the heavy chain sequence [11]. These hydrophobic blocks contain highly repetitive glycine-X $(\mathrm{GX})$ repeats, where $X$ is alanine $(A)(65 \%)$, serine $(S)(23 \%)$ or tyrosine (Y) (9\%) [11]. The exact composition of these hydrophobic regions depends in part on their position in the silk heavy chain.

The GX hydrophobic blocks can be classified into three motifs: (i) a highly repetitive GAGAGS sequence that makes up the bulk of the crystalline regions and is typically found at the start of each block; (ii) a less repetitive sequence, containing hydrophobic and/or aromatic residues GAGAGY, GAGAGV and GAGAGVGY, that forms the semicrystalline regions; and (iii) sequences very similar to (i) except for the presence of an AAS motif, which is usually found at the C-terminal end of each subdomain and may form a sheet-breaking motif (Fig. 1b,c) $[15,16]$. Apart from having a fibroblast growth-promoting peptide at the N-terminal region of the silk heavy chain [17], B. $m o r i$ silk lacks the RGD sequence necessary for integrin-mediated cell attachment.

Overall, the silk heavy chain is of particular importance when designing physically crosslinked silk hydrogels for cell and drug delivery, as it is responsible for 
hydrogel performance. This is because the crystalline regions form physical crosslinks, while the amorphous regions provide network flexibility. These discrete functional domains arise due to the block copolymer arrangement of silk and its ability to adopt a defined secondary structure (for example [16]). Additional chemical crosslinking of silk hydrogels can occur via the reactive amino acids found in the silk (heavy) chain (i.e. the number of these residues are: serine 635 , tyrosine 277 , threonine 47 , glutamic acid 30 and aspartic acid 25) [18]. Therefore, targeting of specific locations within the silk structure is possible because the amino acid sequence of silk is known. For example, serine residues are predominately located within the crystalline domains, while tyrosines are typically found in the non-crystalline domains. The reactive amino acids present in the silk light chain could also be exploited for chemical crosslinking [18]; however, the reverse engineering processes used with the silk cocoons often damage the disulphide linkage between the heavy and light silk chains (detailed below). Therefore, chemistries that target the more robust silk heavy chain are preferred.

The silk primary structure can assume various forms, including random coils, $\beta$ sheets, $\alpha$-helices and turns. The hydrophobic domains can form crystalline anti-parallel $\beta$ sheets joined by hydrogen bonds; these give silk its mechanical strength, while the abundance of these secondary structures determines its stability and mechanical properties $[8,15]$. The formation of the silk secondary structure can be controlled by varying processing conditions, such as water removal, stretching and ions.

One critical consideration in silk chemistry is that the primary structure of native 
silk cannot be faithfully reproduced or engineered via synthetic approaches. The huge protein structure and the high glycine content ( $45.9 \%$ glycine) [11] preclude the use of standard biotechnological approaches to manufacture native B. mori silk.

At its high concentration (up to $35 \% \mathrm{w} / \mathrm{v}$ ) within the silk gland, silk adopts a spherical micelle conformation (100 to $200 \mathrm{~nm}$ in diameter), consisting of a hydrophobic core and a hydrophilic shell formed by the N- and C-terminal domains [19] (Fig. 1a). The $\mathrm{N}$-terminal domain is $\mathrm{pH}$ responsive, so that a drop in $\mathrm{pH}$ in the anterior part of the silk gland results in protonation of the acidic side chains. This, in turn, promotes hydrogen bonding and the transition of silk I (random coils, helices and hydrated $\beta$-strands) to a silk II conformation (i.e. $\beta$-sheets). One potential scenario includes a stabilisation of the silk gel state by $\mathrm{pH}$-induced hydrogen bonding through aggregation of the spherical micelles [20]. The micelles subsequently elongate and align in response to shear forces during the spinning process and the spun silk thread emerges from the head of the silk worm. During the spinning process, the $\beta$-sheet crystals are preferentially aligned parallel to the fibre axis [20]. These $\beta$-sheet crystals are distributed within the amorphous silk matrix but are able to interlock because of partial twisting of the nanofibrils. The interlocking minimises slippage and inhibits crack propagation when a load is applied [21]. Thus, the hierarchal structure of silk, from its unique amino acid composition to its assembly, is responsible for the overall mechanical properties of this biopolymer.

\section{Reverse engineering the silk cocoon}

In the early 1930s the traditional silk processing methodologies were refined and first 
reports emerged for the use of $\mathrm{Na}_{2} \mathrm{CO}_{3}$ for degumming [22] (i.e. removing sericin and waxes, detailed below) and $\mathrm{LiBr}$ [23] to solubelise the silk fibre (Fig. 2). The ability of silkworms to synthesise and store liquid silk has subsequently inspired David Kaplan (Tufts University, MA, USA) and co-workers to use these top-down approach to fully reverse engineered silk cocoons for biomedical applications. An aqueous B. mori silk solution is typically used to generate novel silk formats, including (self-assembling) silk hydrogels. The processing parameters selected for reverse engineering have a direct impact on the resulting silk. A key goal of silk processing is the complete removal of sericin, because sericin is a known inducer of inflammatory responses [2, 24]. Sericin also acts as a "glue", and emerging evidence suggests that it also inhibits the premature conversion of soluble silk (silk I) into gelated and $\beta$-sheet silk conformations [25].

The first step in reverse engineering a silk cocoon is to cut it into small pieces [26]; this contrasts starkly with the treatment of silk for textile applications, where the intact silk thread is required. The silk pieces are then "degummed" by enzymatic methods (i.e. digestion of sericin but not silk) or chemical processing (e.g. alkaline treatment, most typically $\mathrm{Na}_{2} \mathrm{CO}_{3}$ ) to remove sericin. Chemical processing with sodium carbonate is the most common method and typically involves boiling for 20 to 60 minutes (i.e. the degumming time). Complete sericin removal can be achieved within 5 minutes, but this prolonged degumming ( $>5$ minutes) process damages the silk structure, and especially the disulphide linkage between the silk light and heavy chain, as well as the amorphous regions within the silk heavy chain [27]. This damage is seen as an increase in silk polydispersity, a reduction in silk solution conformation from $100 \AA$ (for native silk) to 
$25 \AA$ (measured by small angle neutron scattering) [28] and changes in rheological properties [29]. Boiling times are often empirically selected, but prolonged degumming will ultimately yield a silk solution that takes longer to aggregate (and thus has a longer shelf life) and is easily filter-sterilised due to reduced viscosity. Optimum degumming times have ranged from 20 minutes for the manufacture of silk scaffolds to more than 60 min for the production of nanoparticles [30]. However, extensive degumming ( $>60$ minutes) is expected to adversely affect silk self-assembly, which in turn is detrimental to the formation of physically crosslinked silk hydrogels (i.e. the time for the solution-gel transition is prolonged). Therefore, most studies reported in literature use degumming times of 20 to 60 minutes to create silk stocks (which are then fully reverse engineered, as detailed below).

Following degumming, the pure silk fibres are dried and subsequently dissolved in a chaotropic agent (e.g. lithium bromide, urea, etc.) [26]. For example, silk fibres are dissolved in $\mathrm{LiBr}\left(\right.$ e.g. $9.3 \mathrm{M}$ ) at $60^{\circ} \mathrm{C}$ for up to 4 hours to dissemble the silk secondary and tertiary structure. The resulting viscous solution is then extensively dialysed against water to yield an aqueous silk solution (typically $7 \% \mathrm{w} / \mathrm{v}$ ). Higher silk concentrations (e.g. $20 \% \mathrm{w} / \mathrm{v}$ ) can be achieved by dialysing the silk solution against polyethylene glycol to extract water (note that prolonged dialysis will lead to the formation of a silk hydrogel). During the reverse engineering, the silk does not aggregate and the resulting silk solution is stable at $4^{\circ} \mathrm{C}$ for several months. (Note that silk concentrations higher than $8 \% \mathrm{w} / \mathrm{v}$ will aggregate more easily) (Fig. 2). The reverse engineered silk solution can be used to generate many different silk formats (e.g. films, fibres, scaffolds, (nano)particles, 
etc.) $[1,30]$, including silk hydrogels for drug and cell delivery.

\section{Delivery of therapeutic payloads - key considerations}

During the early stages of drug development, the use of the active pharmaceutical ingredient (i.e. the drug) to study the mechanism of drug action is often sufficient. However, moving a promising active pharmaceutical ingredient into animal models, and ultimately into man, typically requires the "formulation" of the drug into a medicine- - a key mission of pharmaceutical sciences. To complete its journey from bench to bedside, a drug must be evaluated for a number of factors; for example, the most appropriate route of administration (e.g. oral, parenteral, transdermal, subcutaneous, ocular, intra-articular etc.) with the view of using a "local" or "systemic" treatment strategy.

In the context of cancer, targeting a systemically administered drug therapy to the tumour site using a macromolecular carrier approach is desirable, as this will (i) reduce side effects, (ii) increase drug concentrations at the target site and (iii) ultimately modulate the pharmacokinetic characteristics, independent of the physicochemical properties of the therapeutic payload. A macromolecular carrier approach also has the potential to overcome drug resistance mechanisms (e.g. efflux pumps in the plasma membrane) (e.g. [31]). This is because the cell entry mechanisms for a small molecular payload can be changed from passive diffusion across the plasma membrane (i.e. governed by the physicochemical properties of the drug) to a carrier-mediated endocytic uptake mechanism (i.e. uptake governed by the design of the macromolecule and not the payload) (e.g. [31]). Already, today, a number of macromolecular carrier approaches are 
being used in the clinic to deliver small molecular weight anticancer drugs [32], while silk-based systems (i.e. nanoparticles) are in early pre-clinical development [30].

Alternatively, focal (i.e. local) cancer therapy is used clinically for cancer therapy (reviewed in $[33,34])$. Examples include intravesical therapy for bladder cancer, hepatic arterial infusion therapy for hepatocellular carcinoma, brachytherapy of early stage breast cancer and delivery of carmustine by controlled-release polymeric wafers (Gliadel wafers) for the post-resection treatment of high-grade malignant glioma and recurrent glioblastoma multiforme. Anticancer drug delivery systems based on silk films [35-37], foams [38] and self-assembling silk hydrogels [38, 39] are currently showing encouraging results in preclinical animal models of breast cancer and neuroblastoma [33].

Irrespective of the choice of "local" or "systemic" delivery, the therapeutic payload must be sufficiently stable to withstand the conditions of the intended route of administration. For example, orally administered proteins are typically broken down due to the low $\mathrm{pH}$ in the stomach and the presence of proteolytic enzymes in the GI tract; thus, protein drugs are not suitable for an oral route. Furthermore, the delivery system/payload must have the ability to cross biological barriers encountered during administration. The most commonly cited barriers to drug delivery include the gastrointestinal tract, blood-brain barrier and intact skin; although the extent and barrier function various across these system. In deed many more drug delivery barriers exist. For example, macromolecular carriers designed for intracellular activation typically end up in the acidic environment of lysosomes, so their payloads need to be compatible with low 
pH. Payloads accumulating in specific intracellular organelles (e.g. lysosomes) also require the ability to escape and reach their intended site of action (e.g. weakly basic drugs get trapped in lysosomes due to protonation in the acidic environment of the lysosomes, while endosomal/lysosomal escape of genetic material is hindered by the barrier function of the organelle membrane).

Only molecules that meet a specific set of physicochemical properties are able to cross these biological barriers, and many drug candidates fail to meet these criteria. The design of a drug carrier system therefore has to facilitate the delivery of these drugs. For example, with transdermal patch technology, a set of well-known molecular characteristics of the payload are required for successful drug delivery across the skin. By contrast, transiently breaching the barrier function of the skin with microneedles enables the delivery of a much wider spectrum of drugs that, classically, have not been suitable candidates for transdermal delivery. This drug delivery challenge is now being met with a broad range of materials for microneedle design, including silk-based microneedles that are currently in pre-clinical development [40].

While the concepts for delivery active pharmaceutical ingredients are well established, the delivery of living cells as therapies is more challenging because of the need to ensure that the cells maintain their viability and are able to perform their intended functions. Therefore, intense efforts by the tissue engineering community are ongoing to deliver cells and to ensure that the delivered cells can augment or even regenerate the 
target tissues (reviewed [41]). In this context, silk has emerged as a useful biopolymer for the delivery of cell-based therapeutics.

\section{Rationale for using silk for drug and cell delivery}

The attributes of silk typically cited for its use for cell and drug delivery, include (i) biocompatibility, (ii) biodegradability, (iii) mild processing conditions, (iv) protection of the payload and (v) approved use in humans. However, the generic use of the term "biocompatibility" to describe all silks is potentially misleading. First, clinical experience with silk in humans is limited to B. mori silk-based films for wound healing (although with limited public accessible data) and its use as sutures and surgical meshes (a strong track record exists for these latter two). Therefore, novel applications of silk still require careful assessment to ensure patient safety, and this is of particular importance when novel silk formats are being proposed for applications that go beyond current loadbearing applications and topical placement on the skin.

The concept that a material needs to be "fit for its intended purpose" is well established [42], and greater rigor is needed by the scientific community to ensure that silk is not simply labelled as "biocompatible" without supporting evidence for the ultimate intended use.

\section{Silk hydrogel biocompatibility and biodegradation}

Adverse reactions reported for silk (sutures and surgical meshes) in man can be largely attributed to contaminating sericin, as this causes an allergic reaction [24]. Like all non- 
autologous materials, silk elicits a foreign body response following implantation in vivo, but this response is comparable to that induced by the most popular synthetic materials in use today [e.g. poly(lactic-co-glycolic acid), polycaprolactone, polylactic acid etc.] [2].

The two most prominent biocompatibility studies for silk films [43] and scaffolds [44] are often cited as evidence that silk hydrogels are also biocompatible. This is not scientifically rigorous evidence, because the material format, silk processing, implantation site and amount of material transplanted, as well as the animal model used, will all influence biological performance. Therefore, application-oriented biocompatibility studies are important. For example, Michael House (Tufts Medical Center, Boston, MA, USA) and co-workers are making the first inroads into assessing the biocompatibility of silk hydrogels in pregnant rats as a potential therapeutic strategy to prevent preterm birth [45]. Here, sonication-induced silk hydrogels were filled into syringes and injected into the cervixes of pregnant rats (gestational day 13) before the hydrogel had completed the solution-gel transition. Histological assessment of the tissue response at gestational day 17 showed a mild foreign body response similar to that observed with polyglycollic acid and poly(ethylene terephthalate) sutures [45]. This short-term in vivo biocompatibility study was supplemented by in vitro studies with human cervical cells, which showed no up-regulation of inflammatory markers [45]. However, the longer-term effects, such as longitudinal inflammatory responses, biodegradation or impacts on pregnancy (e.g. a shift in the delivery date), are currently unknown. 
One longer-term study (3 months) compared sonication-induced silk hydrogels and collagen type I hydrogels both in vitro and in vivo [46]. Nude mice, at one and two weeks post implantation of silk hydrogels, showed a local inflammatory response but this was substantially less than the response observed with collagen hydrogels. Both the collagen and the silk material attracted neutrophils, eosinophils and macrophages, but these immune cells infiltrated the collagen hydrogel material and subsequently degraded the hydrogel completely within 4 weeks. By contrast, the silk hydrogels showed no infiltration by these cells at 1 or 2 weeks. At week 4 , the inflammation around the silk hydrogel was greatly reduced and the hydrogel had cracks that were populated by spindle shaped cells. At 3 months, no inflammatory cells could be detected in or around the silk hydrogels, but vascularisation was apparent and the spaces in the hydrogel cracks were populated by stromal cells [46]. The use of athymic mice (in the absence of transplanting xenogenic cells) to assess biocompatibility is surprising due to the lack of the adaptive immune system (although this is typically not activated by silk).

In rats, subcutaneously implanted $8 \% \mathrm{w} / \mathrm{v}$ silk hydrogels showed significant hydrogel remodelling at 15 weeks post implantation, resulting in vascularisation and loss of the hydrogel shape [47]. The performance of sonication-induced silk hydrogels was also assessed in healthy brains of adult mice [48]. Silk undergoing the solution-gel transition was stereotaxically injected into the caudate putamen (striatum) and subsequent silk performance was examined using a battery of histological, electrophysiological and behavioural assessments. Flow cytometry of CD45 positive (immune) cells showed a significant increase at 72 hours post silk injection, but the numbers declined at 2 weeks to 
levels comparable to a physiological saline control group. At 4 weeks, the silk or physiological saline injected animals had equivalent numbers of CD45 positive cells when compared with healthy controls [48].

Preliminary studies in healthy rabbits indicated that intravitreal injection of sonication-induced $2 \% \mathrm{w} / \mathrm{v}$ silk hydrogels resulted in a similar acute response at early time points when compared with a vehicle control. At day 30, the silk-treated animals showed a mild inflammation, primarily in the anterior portion of the eye, cornea and vitreous. This response, especially in the vitreous, appeared to be transient and was less frequent and severe at the end point of the study (day 90) [49]. Overall, dedicated biocompatibility studies for silk hydrogels are scarce, but the available data are encouraging [45-49].

The US Pharmacopeia classifies silk (sutures) as non-resorbable. However, this is based on the definition that the material "loses most of its tensile strength within 60 days" post-implantation in vivo. Silk sutures significantly degrade within 1 year, and they are completely resorbed within 2 years [24]. Similar observations have been made for silk surgical meshes. While substantial data exists for the biodegradation of silk films and scaffolds in vivo, only a few studies relate to silk hydrogels. For example, Etienne and coworkers used sonication-induced hydrogels that were preformed ex vivo using a $4 \% \mathrm{w} / \mathrm{v}$ silk solution (40 minute degummed) [46]. Cylindrically shaped $8 \times 6 \mathrm{~mm}$ silk hydrogels were then implanted subcutaneously in Swiss nude mice and the biological response assessed over 3 months by histology [46]. At the end point of this study, fragmentation of 
the silk hydrogel was reported, although the hydrogel maintained its shape and showed signs of vascularisation [46]. However, the exact extent of silk hydrogel degradation was not assessed, nor was the mechanism of vascularisation.

Silk hydrogels $(8 \% \mathrm{w} / \mathrm{v})$ implanted into the hind limb thigh of adult rats showed a time dependent increase in hydrogel pore size and vascularisation over 18 weeks, which was attributed to silk degradation and remodelling [50]. Preliminary studies have assessed the biodegradation of sonication-induced silk hydrogels in healthy brains of adult mice [48]. Based on histological assessment, the $2 \% \mathrm{w} / \mathrm{v}$ silk hydrogel showed a volume reduction of approximately $50 \%$, which was attributed to silk hydrogel biodegradation [48]. (Note, however, that other scenarios are possible, such as hydrogel or tissue contraction).

In vitro studies and mapping of the silk primary sequence to known protease cleavage sites indicates that proteases (e.g. $\alpha$-chymotrypsin, collagenase) and matrix metalloproteinases (MMPs; MMP-1, MMP-2, interstitial collagenase and gelatinase A) are particularly active in silk degradation [51]. Silk is biodegradable due to its susceptibility to (serine/cysteine) proteases (e.g. matrix metalloproteinases, collagenase and $\alpha$-chymotrypsin) and enzyme-catalysed hydrolysis reactions (Fig. 3). In particular, the disulphide bond between the light and heavy chains, as well as the amorphous silk sequences, are highly susceptible to degradation. By contrast, the crystalline regions in the silk heavy chain are substantially more resistant to proteolytic degradation due to reduced chain flexibility and access. Therefore, the $\beta$-sheet content has a protective effect 
on silk degradation both in vitro $[24,43,51-53]$ and in vivo $[43,44]$. The packing geometries also show differences within the crystalline regions, ranging from tight to looser chain packing. The more loosely packed crystalline regions are particularly more susceptible to degradation and are degraded first [53]. Experience with silk films and scaffolds have indicated that the scaffolds degrade faster, most likely due to their increased surface area to mass ratio for proteolytic attack when compared to a monolithic film. One might therefore speculate that silk hydrogels would degrade even faster than silk scaffolds.

Silk sequence alignment indicates 434 and $81 \alpha$-chymotrypsin cleavage sites in the silk heavy chain and light chain, respectively, and 348 and 41 protease XIV cleavage sites, respectively (Fig. 3) [9]. Perhaps counterintuitively, this suggests that $\alpha$ chymotrypsin degrades silk significantly faster than protease XIV. However, experimental evidence clear indicate that protease XIV leads to faster silk hydrogel degradation than $\alpha$-chymotrypsin [51]; similar observations have been reported for other material formats, including silk nanoparticles [9]. Thus, the differences in silk degradation are not governed only by the number of cleavage sites but also depend on enzyme accessibility, the silk format and the secondary structure of the silk.

Protease XIV is a useful proteolytic model enzyme for uncovering some of the fundamentals of silk degradation over short study intervals (e.g. hours to days). Protease XIV first digests the more loosely packed $\beta$-sheets (rather than the densely packed ones). However, protease XIV is a non-mammalian enzyme and thus of limited relevance when 
assessing silk degradation in the biomedical context. Furthermore, protease XIV degradation products yielded both soluble products and nanofibrils (4 nm thick and $80-$ $100 \mathrm{~nm}$ long) that reduced cell viability $\left(\mathrm{IC}_{50} 75 \mu \mathrm{g} / \mathrm{ml}\right.$ ), whereas $\alpha$-chymotrypsin degradation products were not cytotoxic at the tested concentrations $\left(\mathrm{IC}_{50}>225 \mu \mathrm{g} / \mathrm{ml}\right)$ [53]. Overall, these findings emphasise the need to select and apply the most appropriate biocompatibility testing to yield meaningful results.

\section{The challenges with silk}

Silk has many advantages over other (bio)polymers, but it also has limitations. For example, the exact molecular size of silk varies across different B. mori strains [10]. It is therefore important to work with a reliable silk supply chain to ensure product consistency.

B. mori silk is a natural product and thus highly responsive to its environmental conditions during sericulture. This necessitates the supply of high-quality mulberry leaves, performance of pest control monitoring and maintenance of optimum rearing conditions. For example, humidity during silk cocoon spinning impacts the final product. Silk spun in a low-humidity environment shows a low crystallinity due to the limited availability of water vapour for post-spun silk annealing [54]. Humidity can thus impact the mechanical properties of silk. Nonetheless, a potential advantage of silk cocoons spun at low humidity is that the silk can be physically extracted from sericin [54]. This

eliminates the need to boil the silk, which can damage its primary structure and increases the biopolymer's polydispersity. 
Silk hydrogel studies typically use a polydispersed, reverse-engineered silk solution. However, this complicates comparisons across different studies and makes it more challenging to implement quality control measures and, ultimately, clinical translations. A reverse-engineered silk solution has a limited shelf life because the silk will eventually self-assemble, which necessitates the constant supply of fresh silk. Reverse engineering silk is a batch process and difficult to fully automate. This is a challenge for the pharmaceutical industry, where current manufacturing paradigms are shifting to a continuous production process with minimal manual labour.

Although silk has a long track record of human use, adverse reactions to virgin silk sutures have demonstrated that complete sericin removal is critical to achieving biocompatibility in patients [24]. Therefore, appropriate fibre processing is a crucial step to generating hypoallergenic silks. Silk-based medical devices approved for human use demonstrate that it is possible to navigate these challenges and generate silk that complies with Good Manufacturing Practices and licensing requirements.

\section{Silk hydrogels for drug and cell delivery}

In the context of drug delivery, silk hydrogels are exploited for their ability to trap, retain, protect and deliver therapeutics payloads. The selection of the silk processing parameters

(e.g. the amount of silk) and the inclusion of silk hydrogel modification(s) (e.g. drug loaded silk particles, chemical modification of silk, etc.) impact the overall performance. Furthermore, the selection of the payload (e.g. small molecular weight drug, therapeutic 
protein, etc.) and the subsequent environment the silk hydrogel encounters (e.g. vitreous of the eye, tumour microenvironment, etc.) will determine its overall performance (e.g. drug release, biocompatibility, biodegradation etc.). Unlike silk hydrogels used for drug delivery, those used for cell delivery typically serve as a (orphan) cell niche. Therefore fine tuning strategies (e.g. elastic modulus, chemical functional groups, etc.) applied to silk hydrogels typically aim to recapitulate the extracellular matrix (ECM). The ECM, in turn, orchestrates numerous functions, which include (but are not limited to) (stem) cell quiescence, migration, proliferation, self-renewal and differentiation [55]. Thus silk hydrogel modification opens up new possibilities to endow silk with novel functions, including its ability to serve as an ECM mimetic.

Silk lacks integrin binding domains, but the presence of chemically reactive groups at known sites within the silk molecule enables its precise functionalisation at predefined locations [18]. However, unmodified silk hydrogels have also been successfully used for cell culture probably due to the adsorption of serum proteins (e.g. fibronectin) and to the production of endogenous ECM by the cells themselves. Irrespective of their intended use, self-assembling silk hydrogels have excellent injectability due to shear thinning, they show minimal swelling during the solution-gel transition and they typically have good biocompatibility.

Self-healing hydrogels are emerging as useful materials for biomedical applications. However, self-assembling silk hydrogels form strong physical cross-linked networks that are not self-healing. Applying the basic principles of dynamic ligand- 
mediated self-assembly has therefore resulted in the first generation of chemically modified self-healing silks [56]. Furthermore, generating chimeric silks and alloys can endow the resulting materials with novel properties by exploiting recombinant protein technologies to copy nature [57].

Silk hydrogels can be broadly classified into physically [58] and chemically crosslinked systems [59]. For the purpose of this critical analysis, only selected silk hydrogel systems are discussed because others are comprehensively collated in the silk hydrogel literature (for example, $[6,60])$.

\section{Physically crosslinked silk hydrogels}

Physically crosslinked silk hydrogels are particularly promising for drug delivery, tissue engineering and biomedical applications because their formation does not rely on chemical crosslinking. Therefore, fabrication avoids the use of potentially harmful agents such chemical initiators, crosslinkers or UV irradiation. Residual chemicals can leach from chemically crosslinked hydrogels, while UV-based polymerisation techniques are typically incompatible with cell viability or biopharmaceuticals. By contrast, selfassembling silk hydrogels are particularly well suited for biopharmaceuticals and cell delivery because the payload can be added after the silk treatment but prior to the onset of gelation [1]. 
A number of strategies have been explored to generate physically crosslinked silk hydrogels: (i) vortexing [61], (ii) ultrasound [44], (iii) temperature [62], (iv) osmotic stress [62, 63], (v) pH [58], (vi) $\mathrm{CO}_{2}$ acidification [64], (vii) non-solvent induced phase separation [65], (viii) electrical fields [66, 67] and (viii) polymers [68]. The basis for all these hydrogels is the self-assembling behaviour of silk due to the physical entanglements and hydrogen bonding between hydrophobic domains of the silk block copolymer. For many silk hydrogels, a change in secondary structure occurs that typically includes the formation of $\beta$-sheets due to water exclusion [69]. This is exemplified by the addition of highly hydrophilic polyethylene glycol (e.g. PEG300, PEG400), which binds water and results in excluded volume effects [68]. The resulting $\beta$-sheets give rise to strong intermolecular interactions and stabilise the silk hydrogel network, making the hydrogel structure essentially irreversible [69].

Above the critical micelle concentration, silk changes from a random coil to a micellar conformation [28]; in the B. mori silk gland, the high-concentration silk is in a gel-like state. These silk I structures are metastable and undergo weak hydrogen bonding, as well as hydrophobic and electrostatic interactions $[69,70]$. Experimental work with reverse engineered silk confirms the importance of the solution concentration on silk hydrogel self-assembly and structure [70]. Hydrogels derived from low (1\% w/v) and high $(20 \% \mathrm{w} / \mathrm{v})$ concentration reverse-engineered silk solutions showed micellar and fibrillar silk II structures, respectively. Importantly, at high concentrations, fibrillar networks were observed rather than aggregated large globules. Overall, both 
thermodynamics and kinetic components come into play in regulating molecular mobility, hydrophobic/hydrophilic interactions and charge [70].

Mechanistic studies with silk model peptides (GS6: GAGAGS; GS10: GAGAGSGAAS; GY8: GAGAGVGY; GY6: GAGAGY) [71] have provided sequencespecific insights. For example, self-assembly behaviour was dependent on modular motifs, amino acid compositions and concentrations. All peptides, with the exception of GY6, undergo self-assembly, despite differences in secondary structure and concentration dependence. At high concentrations, GS6 and GS10 self-assembled to form disordered aggregates (random coil) to nanofibrils (antiparallel $\beta$-sheet), whereas GY8 selfassembled into forms ranging from disordered aggregates (random coil) to small nanolayer-like structures (antiparallel $\beta$-sheet). At relatively low concentrations, GS6, GS10 and GY8 showed protofilament features. The transition times for the establishment of secondary structure and morphology revealed that the motifs responsible for $\beta$-sheet formation were GS6 $>$ GS10 > GY8 > GY6 [71]. Silk self-assembly has inspired the design of synthetic self-assembling peptides [72-74] and proteins to generate 'silkmimetic' hydrogels (reviewed in [75]).

Reverse engineered silk will eventually form a hydrogel during storage (Fig. 4), but shearing of the solution (i.e. by vortexing, sonication, etc.) speeds up the self-assembling kinetics so that silk hydrogels form within minutes to hours (note that excessive energy input leads to fragmentation of the silk molecule and negatively impacts hydrogel formation [76]). These shear-induced hydrogels transition from a silk I conformation to a 
$\beta$-sheet dominated silk II that contains some inter-chain physical crosslinks [44]. The solution-gel transition kinetics can be further expedited by increasing the silk concentration, solution temperature, concentration of $\mathrm{K}^{+}$ions, acidity (i.e. low $\mathrm{pH}$ ) and energy input, and many of these factors are exploited by selecting a specific manufacturing protocol. For example, $\mathrm{pH}$ can be used as a solution-gel transition trigger. A reduction in the solution $\mathrm{pH}$ to a value close to the silk isoelectric point $(\mathrm{pH} 4.2)$ leads to the rapid formation of hydrogels with extensive $\beta$-sheets [58].

The responsiveness of silk to $\mathrm{pH}$ is governed by its amphiphilic nature and its $\mathrm{pH}$ responsive termini (which enable silk to self-assemble as it progresses along the $\mathrm{pH}$ gradient of the silk gland). The N-terminus of the silk heavy chain is acidic (isoelectric point 4.6) and the C-terminus is basic (isoelectric point 10.5), whereas the C-terminus of the light chain is acidic (isoelectric point 5.1) [60]. The acidic groups become protonated at low $\mathrm{pH}$, leading to a reduced charge-charge repulsion that subsequently allows silk to adopt a more ordered state, with the formation of $\beta$-sheets that exclude water [77]. Another way to activate a $\mathrm{pH}$-mediated trigger is to use high pressure $\mathrm{CO}_{2}$ during silk hydrogel manufacture. At high pressure, $\mathrm{CO}_{2}$ generates carbonic acid, which acidifies the silk solution, changes the hydration shell and reduces volume states of silk. This methods drives gelation within 2 hours, yielding silk hydrogels that are at least 2-fold more mechanically robust than hydrogels generated using traditional $\mathrm{pH}$ approaches [64].

A very different silk hydrogel is formed in response to low electrical DC fields (typically referred to as e-gels) (Fig. 4). E-gels deposit on the positive electrode and 
hydrogel formation is completed within minutes [66]. The electrogelation process is based on local $\mathrm{pH}$ changes that occur due to water electrolysis. The local $\mathrm{pH}$ drops at the positive electrode to a value below the isoelectric point of silk, thereby enabling silk deposition on the electrode, $[67,78]$ as predicted by ion electrodiffusion [78]. Although $\mathrm{pH}$ is also the key driver for the self-assembly of e-gels, their structure and properties differ markedly from other $\mathrm{pH}$ induced silk hydrogels.

Analysis of the secondary structure of e-gels showed a transition from a random coil conformation to a helical conformation, but no changes in $\beta$-sheet content (as typically seen for other silk hydrogels). Reversal of the electric field led to a dissociation of the silk hydrogel and its subsequent formation at the new positive electrode; this process could undergo many repeat cycles [66]. These hydrogels were formed from nanometre-sized, metastable silk micelles [67]. In the presence of a weak electric field, these silk micelles formed larger spherical structures that ranged in size from nanometres to several micrometres. These particles were able to assemble into hydrogels because of screening of the negative surface charge of the silk particles by the low $\mathrm{pH}$ in the vicinity of the positive electrode [67]. The e-gels formed in this way have strong adhesive properties that are typically absent from other self-assembling silk hydrogels. Furthermore, e-gels have outstanding elastic properties and can withstand strains of up to $2,500 \%$. This contrasts sharply with other physically crosslinked silk hydrogels that rely on $\beta$-sheet crystals to stabilise the hydrogel network, as those hydrogels tend to be brittle, cannot undergo long-range displacements and show low elastic behaviour, with plastic deformation typically occurring at strains greater than $10 \%$. Silk e-gels have been 
proposed for a range of biomedical applications (e.g. adhesives for medical devices, sensors, etc.) $[66,67,78]$. However, the need for a DC current could be regarded as a limiting factor.

\section{Chemically crosslinked silk hydrogels}

The beauty of silk is its ability to self-assemble under mild processing conditions; thus, most studies focus on this character. However, chemically crosslinked silk hydrogels have been synthesised using a range of chemistries [60]. For example, using ethylene glycol diglycidyl ether [59] or horseradish peroxidase to crosslink the phenol groups of tyrosine amino acids [79] to produce silk hydrogels that could withstand a shear strain of $100 \%$ and a compressive strain greater than $70 \%$. The stiffness of these silk hydrogels could be fine-tuned to range from 200 to 10,000 Pa by adjusting the silk degumming times from 60 to 10 minutes, respectively. These crosslinked silk hydrogels are elastic and optically clear over the visible wavelength spectrum [79], unlike physically crosslinked gels that typically contain nanocrystalline regions that scatter light. The chemically crosslinked silk hydrogels have subsequently been exposed to low-energy

ultrafast laser pulses to generate complex 3D patterns within the hydrogels that could subsequently be populated with cells and monitored [80]. In a similar set of studies, silk hydrogels were functionalised with phenol red to endow silk with $\mathrm{pH}$ sensing capabilities [81].

However, horseradish peroxidase is immunogenic and complete removal cannot be ascertained. Therefore, alternative crosslinkers are being explored. For example, 
riboflavin (vitamin B2), a photoactive crosslinker, has also been used to generate in situ crosslinked silk corneal prostheses aimed at improving visual acuity [82].

\section{Examples of silk hydrogels for drug and cell delivery}

Silk hydrogels have been studied for a broad range of biomedical applications, including cell and drug delivery. Self-assembling silk hydrogels, in particular, are ideally suited for these applications (for the reasons detailed above). The following is a critical assessment of a few selected examples.

Delivery of small molecular weight anticancer drugs using self-assembling silk hydrogels

Self-assembling silk hydrogels have been developed for breast cancer focal therapy and subjected to pre-clinical testing [39] (Fig. 5). The reverse engineered silk solution was exposed to sonication cycles, spiked with doxorubicin and subsequently filled into syringes to complete the solution-gel transition. In vitro doxorubicin release from silk hydrogels over 4 weeks showed no burst release but could be fine-tuned: the fastest release was observed for $2 \% \mathrm{w} / \mathrm{v}$ and slowest for $6 \% \mathrm{w} / \mathrm{v}$ silk hydrogels $(17 \%$ and $27 \%$ cumulative drug release, respectively) [39]. The strong charge-dependent drug-silk interaction appears to be a key driver for the loading and release profiles of doxorubicin (a weakly basic drug) [31, 37]. Therefore, increasing the silk content (i.e. from 2 to $6 \%$ w/v) significantly slowed the drug release. 
The silk secondary structure is also an important factor regulating drug release, as a high $\beta$-sheet content gives a slower doxorubicin release from model silk films [37]. The hydrophobic crystalline domains therefore conceivably may provide a "binding" pocket for the hydrophobic doxorubicin; however, direct experimental proof for this binding is currently lacking. Increasing the degumming time from 20 to 45 minutes results in a reduction in the silk molecular weight but has minimal impact on the crystalline domains, which appear to be critical for the silk-doxorubicin interaction. Not surprisingly, then, degumming time did not affect doxorubicin release from silk hydrogels [39].

All doxorubicin loaded silk hydrogels showed excellent in vitro anticancer activity in a human breast cancer relapse model. Therefore, the anticancer activity of doxorubicin loaded silk hydrogels was assessed in an orthotopic triple-negative human breast cancer model (Fig. 5e). Doxorubicin-loaded silk hydrogels injected next to wellestablished breast tumours resulted in complete tumour regression in 2/5 animals and a significantly reduced tumour burden and metastasis in all other animals (Fig. 5e). By contrast, animals dosed intravenously with the equivalent amount of doxorubicin showed only a marginal antitumour effect and significantly greater cardiotoxicity. Thus, drugloaded silk hydrogels showed a promising antitumour response in this pre-clinical animal model [39] and outperformed a PEG-heparin hydrogel system assessed in a parallel study using the same cancer model and doxorubicin loading [83]. A similar set of studies examined self-assembling silk hydrogels loaded with vincristine and tested in an orthotopic neuroblastoma mouse model [38]. This focal therapy was also able to improve survival; these hydrogels performed similarly to drug-loaded silk foams, which 
significantly changed vincristine pharmacokinetics. Focal therapy resulted in very high drug tumour concentrations (up to 1,000-fold greater than for iv-dosed animals) and increased plasma concentrations when compared to the iv-dosed vincristine [38].

\section{Delivery of biologics using self-assembling silk hydrogels}

Physically crosslinked silk hydrogels are well suited for injection because they show shear thinning. Furthermore, silk also has a remarkable ability to stabilise therapeutic proteins (detailed above, reviewed in $[84,85]$ ). Thus, silk hydrogels not only deliver a payload, but they also protect their cargo. Self-assembling silk hydrogels have been examined for their ability to deliver bevacizumab (a monoclonal antibody of antivascular endothelial growth factor) for the treatment of age-related macular degeneration [49]. Silk cocoons were degummed (no details provided) and reverse engineered. Bevacizumab was added to the silk solution at a standard dose $(1.25 \mathrm{mg} / 50 \mu \mathrm{lof} 2 \% \mathrm{w} / \mathrm{v}$ silk solution) and a high dose $(5 \mathrm{mg} / 50 \mu \mathrm{l}$ of $2 \% \mathrm{w} / \mathrm{v}$ silk solution) and subsequently sonicated to induce gel formation. The sonication was performed at a sufficiently low energy to minimise sample heating and to conserve biological activity of bevacizumab. In vitro release studies showed an initial burst release of the drug during the first 10 days for all samples. The highest initial drug release occurred with the commercial preparation, but this release subsequently fell below the level of detection within 33 days. By contrast, drug release from the silk hydrogels was more sustained over 90 days, with bevacizumab levels higher for the high dose samples than for the standard dose ones [49]. 
Next, drug release was assessed in healthy rabbits, which underwent intravitreal injection with $50 \mu \mathrm{l}$ of either the commercial preparation, the standard loaded or the high loaded silk hydrogel preparation [49]. Tracking bevacizumab levels in the blood plasma revealed similar in vivo kinetics to those observed in vitro. Drug levels in the vitreous and aqueous humour at extended times were substantially higher for animals dosed with both silk hydrogel preparations than with the commercial preparation. Pharmacokinetic parameters indicated similar relative bioavailability of the commercial and silk hydrogel bevacizumab (i.e. area under the curve 3,300 and $2,520 \mu \mathrm{g} / \mathrm{ml} \times$ day), but the terminal half-life for both silk hydrogel preparations was 2 to 3 -fold higher. Optical assessment of the silk hydrogels indicated a 30 to $75 \%$ volume reduction, which was interpreted as silk biodegradation (but could also be due to hydrogel shrinkage, or a combination of both). The intravitreal route of administration remains a major challenge because the hydrogel sits in the vitreous [49] and obstructs the optical light path, thereby obscuring vision. Furthermore, the observed burst release for the silk hydrogels requires more optimisation, but similar results have been reported for other monoclonal antibodies and silk delivery systems [86].

\section{(Stem) cell delivery using self-assembling silk hydrogels}

The excellent material properties of silk have supported the development of various hydrogel systems for soft and hard tissue engineering applications (reviewed in [87, 88]). For example, pancreatic islet transplantation is challenging because of the functional decline and reduced viability of the islets during the peritransplantation period. Therefore, self-assembling silk hydrogels $(1.2 \% \mathrm{w} / \mathrm{v})$ were assessed as a potential 
delivery system [47]. Silk was vortexed, and prior to the completion of the solution-gel transition, the silk preparation was loaded with cells. Three treatment groups were assessed: (i) pelleted pancreatic islets (i.e. no delivery matrix), (ii) pancreatic islets embedded in silk and (iii) pancreatic islets with mesenchymal stem cells (MSCs) embedded in silk. Following the completion of the solution-gel transition, diabetic mice were surgically implanted with a graft in the epidermal fat pad, and the grafts were subjected to functional tests (i.e. blood glucose monitoring and intraperitoneal glucose tolerance test).

Silk hydrogels containing marginal pancreatic islets grafts performed best and were able to control blood glucose within 4 days, whereas pelleted pancreatic islets required 15 days and silk hydrogels loaded with both pancreatic islets and MSCs required 9 days. The silk hydrogel was noted to induce expression of vascular endothelial growth factor (VEGF), and VEGF, in turn, could improve graft survival and function. The transplanted MSCs further supported graft function via trophic and angiogenic factor expression, as demonstrated by minimal islet grafts that were able to control glucose levels in 3 out of 4 mice. However, the minimal islet grafts were not able to control glucose when transplanted only with silk or as pelleted pancreatic islets only (Fig. 6) [47].

Although MSCs supported islet function, they also resulted in an unintended outcome: at the end of the study, the transplanted MSCs (day 42) had formed new ossicles [47] indicating that the silk hydrogel matrix was not able to maintain MSCs in a 
multipotent state but instead had inadvertently supported osteogenic differentiation. Therefore, silk hydrogels require fine-tuning to direct MSC lineage commitment, and chemical modification of silk hydrogels [89], the inclusion of growth factors and the adjustment of physical properties [90] will be key parameters that will control MSC behaviour.

Note that silk hydrogels have been used for bone tissue engineering applications (reviewed in [91]), but the hydrogels tend to be brittle. Consequently, the inclusion of silk fibres has emerged as a valuable strategy [92] to improve mechanical strength while serving as a physical cue for MSC differentiation.

\section{Future Perspective}

\section{Printing silk hydrogels}

A range of printing technologies has been used in combination with silk to print: (i) liquid silk [93], (ii) pre-gelled (recombinant) silks [94], (iii) silk-based bioinks [95] and (iv) chemically modified silks [96]. Stable silk structures are created using both physical and chemical crosslinking. For example, reverse engineered silk has been used as a carrier material and matrix for a broad range of applications, including sensing, therapeutics and regenerative medicine [93]. The liquid silk was doped with the active component (e.g. model drugs, sensors, nanoparticles) and subsequently printed onto a range of substrates (e.g. paper, petri dishes, gloves, etc.). The deposited silk was allowed to self-assemble on the respective surface and assessed for functionality: gold nanoparticle-doped printed silk arrays could be selectively heated, bone morphogenic 
protein doped silk could differentiate MSCs into osteoblasts and IgG functionalised silk sensors on gloves could detect bacterial contamination. While these applications demonstrate the versatility of silk, it remains to be seen if a similar diversity can also be achieved with silk hydrogels (note that actually no silk hydrogel was formed in this study [93]). The suitability of self-assembled silk hydrogels, as well as chemically cross linked polymeric networks, for three dimensional printing has been demonstrated with reverse engineered silk [97]. For the self-assembling systems, silk was sonicated and then the cells were added to the liquid silk, which was allowed to complete the solution-gel transition and subsequently extruded under pressure. The resulting three-dimensional constructs could be readily manufactured to a $500 \mu \mathrm{m}$ resolution and supported cell viability [97].

Others have developed liquid bioinks that self-assemble after printing. Here, anionic polyglutamic acid and cationic polylysine modified silks were synthesised, the $\mathrm{pH}$ adjusted to 5.5 and the material sequentially printed to generate silk arrays. Through additive manufacturing, these striated silk arrays were populated with E. coli cells [96]. In other studies, mixtures of silk, gelatine and glycerol have been optimised and subsequently used to print complex three-dimensional structures. Here, the gelatine served as a bulking material and a thermoresponsive switch. The mixture was melted and extruded at $37^{\circ} \mathrm{C}$ to achieve Newtonian like flow characteristics and deposited on a surface at $20^{\circ} \mathrm{C}$ to $25^{\circ} \mathrm{C}$. The structures were stable but required immersion in a glycerol bath to induce $\beta$-sheets to render the printed constructs water insoluble [95]. These types 
of studies are promising, but require further work because the glycerol immersion bath is incompatible with the in situ printing of cells.

Chemical crosslinking has also been piloted; for example, combining silk with alginate and horseradish peroxidase allowed the development of a two-step cross-linking protocol [98]. First, the mixture was printed into a $\mathrm{CaCl}_{2}$ bath to induce ionic cross linking of alginate; the alginate network immobilised the silk and horseradish peroxidase. Next, addition of hydrogen peroxide catalysed the chemical cross-linking of tyrosine residues within the silk sequence. Addition of sodium citrate dismantled the ionic crosslinks of the alginate and subsequent washing removed the sacrificial alginate from the system [98].

Overall, tremendous opportunities exist for the exploitation of silk for printing applications, as demonstrated by the developments reported over the past 5 years.

\section{Summary}

This review provides a detailed background of silk and its many facets, and it summarises recent developments in the production and use of self-assembling silk hydrogels. In it, we have addressed common pitfalls and misconceptions while identifying emerging opportunities. Of course, silk has its limits, and we must recognise these and build on new insights.

\section{Executive Summary}




\section{Silk}

- Numerous different silks exist in nature which are produced by many different animals, including, but not limited to, spiders and silkworms.

- B. mori is the domesticated silkworm which is used commercially to produce silk on an industrial scale typically for the textile industry.

- B. mori silk is approved for human clinical use for load bearing applications in its fibre format (i.e. sutures and surgical mesh) and as a wound dressing (i.e. a silk sponge attached to a silicon film, approved in China only).

\section{Biocompatibility and biodegradation}

- Silk is susceptible to proteolysis leading to silk biodegradation. The rate and extend is dependent on many factors including the silk format, secondary structure and processing history as well as the implantation site in vivo.

- Silk is demonstrating excellent biocompatibility across a range of applications. This trend appears to be robust from mice to man.

\section{Silk hydrogels}

- Many hydrogels exploit the endogenous self-assembly of silk. The underlying principle is typically based on physical crosslinking due to exposure of crystallisable silk sequences.

- Chemical cross linking of silk yields hydrogels which are significantly more elastic than those formed by physical cross linking. Due to the absence of 
nanocrystallites, chemical cross linked hydrogels are optical transparent to visible light.

- Silk hydrogels are emerging as promising substrates for drug and cell delivery applications, especially those systems exploiting the self-assembly behaviour of silk.

\section{Additional Information}

The author declares no competing interests.

\section{References}

[1] Seib FP, Kaplan DL. Silk for drug delivery applications: Opportunities and challenges. Israel J. Chem. 53(9-10), 756-66 (2013).

[2] Thurber AE, Omenetto FG, Kaplan DL. In vivo bioresponses to silk proteins. Biomaterials 71, 145-57 (2015).

[3] Jewell M, Daunch W, Bengtson B, Mortarino E. The development of seri(r) surgical scaffold, an engineered biological scaffold. Ann. N.Y. Acad. Sci. 1358, 44-55 (2015).

[4] Karp N, Choi M, Kulber DA, et al. SERI Surgical Scaffold in 2-stage breast reconstruction: 2-year data from a prospective, multicenter trial. Plast. Reconstr. Surg. Glob. Open. 5(5), e1327 (2017). 
[5] Zhang W, Chen L, Chen J, et al. Silk fibroin biomaterial shows safe and effective wound healing in animal models and a randomized controlled clinical trial. Adv. Healthc. Mater. 6(10), (2017).

[6] Floren M, Migliaresi C, Motta A. Processing techniques and applications of silk hydrogels in bioengineering. J. Funct. Biomater. 7(3), pii: E26 (2016). ** Excellent review summarising the current processing techniques to manufacture silk hydrogels.

[7] Porter D, Guan J, Vollrath F. Spider silk: Super material or thin fibre? Adv. Mater. 25(9), 1275-9 (2013).

[8] Vollrath F, Porter D. Silks as ancient models for modern polymers. Polymer 50(24), 5623-32 (2009).

[9] Wongpinyochit T, Johnston BF, Seib FP. Degradation behavior of silk nanoparticles enzyme responsiveness. ACS Biomater. Sci. Eng., 4(3), 942-951 (2018).

[10] Manning RF, Gage LP. Internal structure of the silk fibroin gene of bombyx mori. Ii. Remarkable polymorphism of the organization of crystalline and amorphous coding sequences. J. Biol. Chem. 255(19), 9451-7 (1980).

[11] Zhou CZ, Confalonieri F, Jacquet M, et al. Silk fibroin: Structural implications of a remarkable amino acid sequence. Proteins 44(2), 119-22 (2001).

[12] Zhou CZ, Confalonieri F, Medina N, et al. Fine organization of bombyx mori fibroin heavy chain gene. Nucleic Acids Res. 28(12), 2413-9 (2000). 
[13] Yamaguchi K, Kikuchi Y, Takagi T, et al. Primary structure of the silk fibroin light chain determined by cdna sequencing and peptide analysis. J. Mol. Biol. 210(1), 127-39 (1989).

[14] Tanaka K, Kajiyama N, Ishikura K, et al. Determination of the site of disulfide linkage between heavy and light chains of silk fibroin produced by bombyx mori. Biochim. Biophys. Acta 1432(1), 92-103 (1999).

[15] Asakura T, Okushita K, Williamson MP. Analysis of the structure of bombyx mori silk fibroin by nmr. Macromolecules 48(8), 234557 (2015).

[16] Asakura T, Ohgo K, Ishida T, et al. Possible implications of serine and tyrosine residues and intermolecular interactions on the appearance of silk i structure of bombyx mori silk fibroin-derived synthetic peptides: High-resolution 13c crosspolarization/magic-angle spinning nmr study. Biomacromolecules 6(1), 468-74 (2005).

[17] Yamada H, Igarashi Y, Takasu Y, Saito H, Tsubouchi K. Identification of fibroinderived peptides enhancing the proliferation of cultured human skin fibroblasts. Biomaterials 25(3), 467-72 (2004).

[18] Murphy AR, Kaplan DL. Biomedical applications of chemically-modified silk fibroin. J. Mater. Chem. 19(36), 6431-42 (2009).

[19] Jin HJ, Kaplan DL. Mechanism of silk processing in insects and spiders. Nature 424(6952), 1057-61 (2003). 
[20] Werner V, Meinel L. From silk spinning in insects and spiders to advanced silk fibroin drug delivery systems. Eur. J. Pharm. Biopharm. 97(Pt B), 392-9 (2015).

[21] Xu G, Gong L, Yang Z, Liu XY. What makes spider silk fibers so strong? From molecular-crystallite network to hierarchical network structures. Soft Matter 10(13), 2116-23 (2014).

[22] Myers LD, Stegemeyer LA. Process of degumming silk. Patent 1896494. USA: Twitchell Process Company; 1933.

[23] Wilhelm I, Herbert M. Solution of silk fibroin and the wastes thereof. Patent 1913487. USA: IG Farbenindustrie AG; 1933.

[24] Altman GH, Diaz F, Jakuba C, et al. Silk-based biomaterials. Biomaterials 24(3), 401-16 (2003).

[25] Kwak HW, Ju JE, Shin M, Holland C, Lee KH. Sericin promotes fibroin silk i stabilization across a phase-separation. Biomacromolecules 18(8), 2343-9 (2017).

[26] Rockwood DN, Preda RC, Yucel T, et al. Materials fabrication from bombyx mori silk fibroin. Nat. Protoc. 6(10), 1612-31 (2011). ** Protocol summarising the manufacture of novel silk formats from reverse engineered silk

[27] Wray LS, Hu X, Gallego J, et al. Effect of processing on silk-based biomaterials: Reproducibility and biocompatibility. J. Biomed. Mater. Res. B Appl. Biomater. 99(1), 89-101 (2011). 
[28] Greving I, Dicko C, Terry A, Callow P, Vollrath F. Small angle neutron scattering of native and reconstituted silk fibroin. Soft Matter 6(18), 4389-95 (2010).

[29] Holland C, Terry A, Porter D, Vollrath F. Natural and unnatural silks. Polymer 48(12), 3388-92 (2007). ** Excellent critical review about silk and the impact of reverse engineering on silk performance.

[30] Seib FP. Silk nanoparticles—an emerging anticancer nanomedicine. AIMS Bioengineering 4(2), 239-58 (2017).

[31] Seib FP, Jones GT, Rnjak-Kovacina J, Lin Y, Kaplan DL. Ph-dependent anticancer drug release from silk nanoparticles. Adv. Healthc. Mater. 2(12), 1606-11 (2013).

[32] Shi J, Kantoff PW, Wooster R, Farokhzad OC. Cancer nanomedicine: Progress, challenges and opportunities. Nat. Rev. Cancer 17(1), 20-37 (2017).

[33] Harris J, Klonoski SC, Chiu B. Clinical considerations of focal drug delivery in cancer treatment. Curr. Drug Deliv. 14(5), 588-96 (2017).

[34] Wolinsky JB, Colson YL, Grinstaff MW. Local drug delivery strategies for cancer treatment: Gels, nanoparticles, polymeric films, rods, and wafers. J. Control. Release 159(1), 14-26 (2012).

[35] Chiu B, Coburn J, Pilichowska M, et al. Surgery combined with controlled-release doxorubicin silk films as a treatment strategy in an orthotopic neuroblastoma mouse model. Br J Cancer 111(4), 708-15 (2014). 
[36] Seib FP, Coburn J, Konrad I, et al. Focal therapy of neuroblastoma using silk films to deliver kinase and chemotherapeutic agents in vivo. Acta Biomater. 20, 32-8 (2015).

[37] Seib FP, Kaplan DL. Doxorubicin-loaded silk films: Drug-silk interactions and in vivo performance in human orthotopic breast cancer. Biomaterials 33(33), 8442-50 (2012).

[38] Coburn J, Harris J, Zakharov AD, et al. Implantable chemotherapy-loaded silk protein materials for neuroblastoma treatment. Int. J. Cancer 140(3), 726-35 (2017).

[39] Seib FP, Pritchard EM, Kaplan DL. Self-assembling doxorubicin silk hydrogels for the focal treatment of primary breast cancer. Adv. Funct. Mater. 23(1), 58-65 (2013). * First use of self-assembling silk hydrogels for anticancer drug delivery.

[40] Tsioris K, Raja WK, Pritchard EM, et al. Fabrication of silk microneedles for controlled-release drug delivery. Adv. Funct. Mater. 22(2), 330-5 (2012).

[41] Mao AS, Mooney DJ. Regenerative medicine: Current therapies and future directions. Proc. Natl. Acad. Sci. U S A 112(47), 14452-9 (2015).

[42] Williams DF. On the mechanisms of biocompatibility. Biomaterials 29(20), 2941-53 (2008).

[43] Meinel L, Hofmann S, Karageorgiou V, et al. The inflammatory responses to silk films in vitro and in vivo. Biomaterials 26(2), 147-55 (2005). 
[44] Wang X, Kluge JA, Leisk GG, Kaplan DL. Sonication-induced gelation of silk fibroin for cell encapsulation. Biomaterials 29(8), 1054-64 (2008). * First report of sonication-induced self-assembling silk hydrogels.

[45] Critchfield AS, McCabe R, Klebanov N, et al. Biocompatibility of a sonicated silk gel for cervical injection during pregnancy: In vivo and in vitro study. Reprod. Sci. 21(10), 1266-73 (2014).

[46] Etienne O, Schneider A, Kluge JA, et al. Soft tissue augmentation using silk gels: An in vitro and in vivo study. J. Periodontol. 80(11), 1852-8 (2009). * First report of the biological performance of self-assembling silk hydrogels in mice.

[47] Hamilton DC, Shih HH, Schubert RA, et al. A silk-based encapsulation platform for pancreatic islet transplantation improves islet function in vivo. J. Tissue Eng. Regen. Med. 11(3), 887-95 (2017).

[48] Fernandez-Garcia L, Mari-Buye N, Barios JA, et al. Safety and tolerability of silk fibroin hydrogels implanted into the mouse brain. Acta Biomater. 45, 262-75 (2016).

[49] Lovett ML, Wang X, Yucel T, et al. Silk hydrogels for sustained ocular delivery of anti-vascular endothelial growth factor (anti-vegf) therapeutics. Eur. J. Pharm.

Biopharm. 95(Pt B), 271-8 (2015). * First report of the biological performance of selfassembling silk hydrogels for occular drug delivery in animals.

[50] Leng X, Liu B, Su B, et al. In situ ultrasound imaging of silk hydrogel degradation and neovascularization. J. Tissue Eng. Regen. Med. 11(3), 822-30 (2017). 
[51] Brown J, Lu CL, Coburn J, Kaplan DL. Impact of silk biomaterial structure on proteolysis. Acta Biomater. 11, 212-21 (2015).

[52] Horan RL, Antle K, Collette AL, et al. In vitro degradation of silk fibroin. Biomaterials 26(17), 3385-93 (2005).

[53] Numata K, Cebe P, Kaplan DL. Mechanism of enzymatic degradation of beta-sheet crystals. Biomaterials 31(10), 2926-33 (2010).

[54] Boulet-Audet M, Holland C, Gheysens T, Vollrath F. Dry-spun silk produces nativelike fibroin solutions. Biomacromolecules 17(10), 3198-204 (2016).

[55] Prewitz M, Seib FP, Pompe T, Werner C. Polymeric biomaterials for stem cell bioengineering. Macromol. Rapid Commun. 33(17), 1420-31 (2012).

[56] Shi L, Wang F, Zhu W, et al. Self-healing silk fibroin-based hydrogel for bone regeneration: Dynamic metal-ligand self-assembly approach. Adv. Funct. Mater. 27(37), 1700591 (2017).

[57] Price R, Poursaid A, Ghandehari H. Controlled release from recombinant polymers. J. Control. Release 190, 304-13 (2014).

[58] Ayub ZH, Arai M, Hirabayashi K. Mechanism of the gelation of fibroin solution. Biosci. Biotech. Biochem. 57(11), 1910-2 (1993).

[59] Min S, Nakamura T, Teramoto A, Abe K. Preparation and characterization of crosslinked porous silk fibroin gel. Fiber 54(2), 85-92 (1998). 
[60] Kapoor S, Kundu SC. Silk protein-based hydrogels: Promising advanced materials for biomedical applications. Acta Biomater. 31, 17-32 (2016).

[61] Yucel T, Cebe P, Kaplan DL. Vortex-induced injectable silk fibroin hydrogels. Biophys. J. 97(7), 2044-50 (2009).

[62] Kim UJ, Park J, Li C, et al. Structure and properties of silk hydrogels. Biomacromolecules 5(3), 786-92 (2004).

[63] Ribeiro M, de Moraes MA, Beppu MM, Monteiro FJ, Ferraz MP. The role of dialysis and freezing on structural conformation, thermal properties and morphology of silk fibroin hydrogels. Biomatter. 4, e28536 (2014).

[64] Floren ML, Spilimbergo S, Motta A, Migliaresi C. Carbon dioxide induced silk protein gelation for biomedical applications. Biomacromolecules 13(7), 2060-72 (2012).

[65] Kasoju N, Hawkins N, Pop-Georgievski O, Kubies D, Vollrath F. Silk fibroin gelation via non-solvent induced phase separation. Biomater. Sci. 4(3), 460-73 (2016).

[66] Leisk GG, Lo TJ, Yucel T, Lu Q, Kaplan DL. Electrogelation for protein adhesives. Adv. Mater. 22(6), $711-5$ (2010). * First report of e-gels in the literature.

[67] Lu Q, Huang Y, Li M, et al. Silk fibroin electrogelation mechanisms. Acta Biomater. 7(6), 2394-400 (2011).

[68] Wang X, Partlow B, Liu J, et al. Injectable silk-polyethylene glycol hydrogels. Acta Biomater. 12, 51-61 (2015). 
[69] Matsumoto A, Chen J, Collette AL, et al. Mechanisms of silk fibroin sol-gel transitions. J. Phys. Chem. B 110(43), 21630-8 (2006).

[70] Lu Q, Zhu H, Zhang C, et al. Silk self-assembly mechanisms and control from thermodynamics to kinetics. Biomacromolecules 13(3), 826-32 (2012).

[71] Mengjia M, Zhong J, Li W, et al. Comparison of four synthetic model peptides to understand the role of modular motifs in the self-assembly of silk fibroin. Soft Matter 9(47), 11325-33 (2013).

[72] Guo H, Zhang J, Xu T, et al. The robust hydrogel hierarchically assembled from a ph sensitive peptide amphiphile based on silk fibroin. Biomacromolecules 14(8), 2733-8 (2013).

[73] Nieuwland M, van Gijzel N, van Hest JC, Lowik DW. The influence of amino acid sequence on structure and morphology of polydiacetylene containing peptide fibres. Soft Matter 11(7), 1335-44 (2015).

[74] Zhan J, Cai Y, Ji S, et al. Supramolecular silk from a peptide hydrogel. Mater Chem Front 1, 911-5 (2017).

[75] DiMarco RL, Heilshorn SC. Multifunctional materials through modular protein engineering. Adv Mater 24(29), 3923-40 (2012).

[76] Samal SK, Kaplan DL, Chiellini E. Ultrasound sonication effects on silk fibroin protein. Macromol. Mater. Eng. 298(11), 1201-8 (2013). 
[77] He YX, Zhang NN, Li WF, et al. N-terminal domain of bombyx mori fibroin mediates the assembly of silk in response to ph decrease. J. Mol. Biol. 418(3-4), 197-207 (2012).

[78] Kojic N, Panzer MJ, Leisk GG, et al. Ion electrodiffusion governs silk electrogelation. Soft Matter 8(26), 6897-905 (2012).

[79] Partlow BP, Hanna CW, Rnjak-Kovacina J, et al. Highly tunable elastomeric silk biomaterials. Adv. Funct. Mater. 24(29), 4615-24 (2014).

[80] Applegate MB, Coburn J, Partlow BP, et al. Laser-based three-dimensional multiscale micropatterning of biocompatible hydrogels for customized tissue engineering scaffolds. Proc. Natl. Acad. Sci. U S A 112(39), 12052-7 (2015).

[81] Sundarakrishnan A, Herrero Acero E, Coburn J, et al. Phenol red-silk tyrosine crosslinked hydrogels. Acta Biomater. 42, 102-13 (2016).

[82] Applegate MB, Partlow BP, Coburn J, et al. Photocrosslinking of silk fibroin using riboflavin for ocular prostheses. Adv. Mater. 28(12), 2417-20 (2016).

[83] Seib FP, Tsurkan M, Freudenberg U, Kaplan DL, Werner C. Heparin-modified polyethylene glycol microparticle aggregates for focal cancer chemotherapy. ACS Biomater. Sci. Eng. 2(12), 2287-93 (2016).

[84] Li AB, Kluge JA, Guziewicz NA, Omenetto FG, Kaplan DL. Silk-based stabilization of biomacromolecules. J. Control. Release 219, 416-30 (2015). 
[85] Pritchard EM, Dennis PB, Omenetto FG, Naik RR, Kaplan DL. Physical and chemical aspects of stabilization of compounds in silk. Biopolymers 97(6), 479-98 (2012).

[86] Guziewicz N, Best A, Perez-Ramirez B, Kaplan DL. Lyophilized silk fibroin hydrogels for the sustained local delivery of therapeutic monoclonal antibodies. Biomaterials 32(10), 2642-50 (2011).

[87] Abbott RD, Kaplan DL. Engineering biomaterials for enhanced tissue regeneration. Curr. Stem Cell Reports 2(2), 140-6 (2016).

[88] Abbott RD, Kimmerling EP, Cairns DM, Kaplan DL. Silk as a biomaterial to support long-term three-dimensional tissue cultures. ACS Appl. Mater. Interfaces 8(34), 21861-8 (2016).

[89] Calabrese R, Kaplan DL. Silk ionomers for encapsulation and differentiation of human mscs. Biomaterials 33(30), 7375-85 (2012).

[90] Floren M, Bonani W, Dharmarajan A, et al. Human mesenchymal stem cells cultured on silk hydrogels with variable stiffness and growth factor differentiate into mature smooth muscle cell phenotype. Acta Biomater. 31, 156-66 (2016).

[91] Melke J, Midha S, Ghosh S, Ito K, Hofmann S. Silk fibroin as biomaterial for bone tissue engineering. Acta Biomater. 31, 1-16 (2016). 
[92] Yodmuang S, McNamara SL, Nover AB, et al. Silk microfiber-reinforced silk hydrogel composites for functional cartilage tissue repair. Acta Biomater. 11, 27-36 (2015).

[93] Tao H, Marelli B, Yang M, et al. Inkjet printing of regenerated silk fibroin: From printable forms to printable functions. Adv. Mater. 27(29), 4273-9 (2015).

[94] Schacht K, Jungst T, Schweinlin M, et al. Biofabrication of cell-loaded 3d spider silk constructs. Angew. Chem. Int. Ed. Engl. 54(9), 2816-20 (2015).

[95] Rodriguez MJ, Brown J, Giordano J, et al. Silk based bioinks for soft tissue reconstruction using 3-dimensional (3d) printing with in vitro and in vivo assessments. Biomaterials 117, 105-15 (2017).

[96] Suntivich R, Drachuk I, Calabrese R, Kaplan DL, Tsukruk VV. Inkjet printing of silk nest arrays for cell hosting. Biomacromolecules 15(4), 1428-35 (2014).

[97] Das S, Pati F, Choi YJ, et al. Bioprintable, cell-laden silk fibroin-gelatin hydrogel supporting multilineage differentiation of stem cells for fabrication of three-dimensional tissue constructs. Acta Biomater. 11, 233-46 (2015).

[98] Compaan AM, Christensen K, Huang Y. Inkjet bioprinting of 3d silk fibroin cellular constructs using sacrificial alginate. ACS Biomater. Sci. Eng. 3(8), 1519-26 (2017). 
A
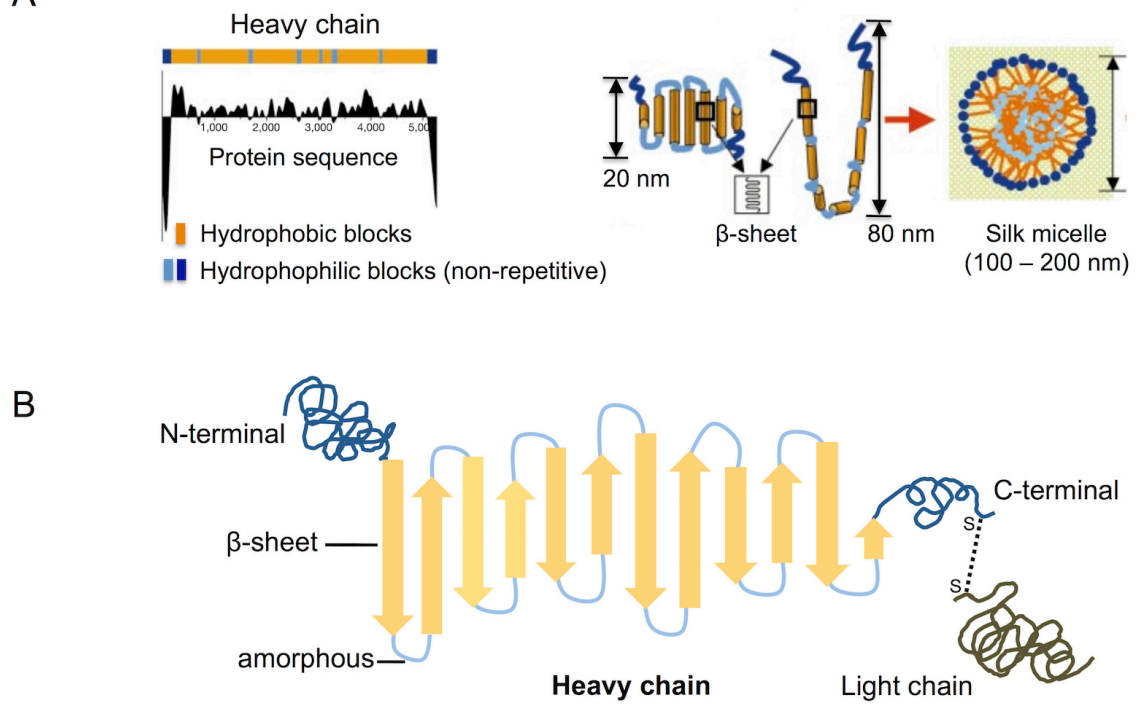

C

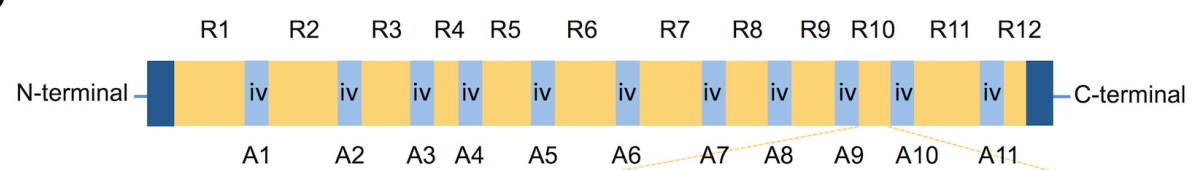

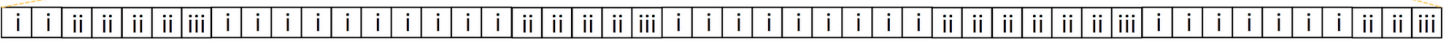

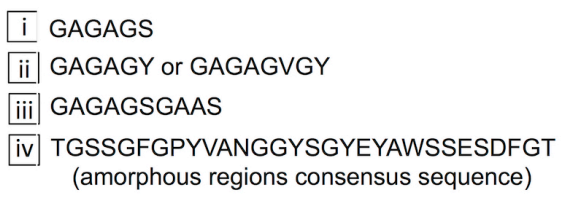

Figure 1. Silk structure of Bombyx mori. (A) Solution conformation of silk. Hydrophobicity pattern of the heavy chain with possible chain folding and micelle assembly of silk in water. (B) Two dimensional silk schematic. (C) Primary structure of the silk heavy chain. R01 to R12 and A01 to A11 represent the arrangement of 12 repetitive and 11 amorphous regions, respectively. The approximate amino acid sequence of the R10 is shown by combination of sequences of $\mathrm{i}$, ii and iii. (Panel A adapted with 
permission from [19]. Panel B adapted with permission from [9] and panel C adapted with permission from [16], Copyright American Chemical Society).

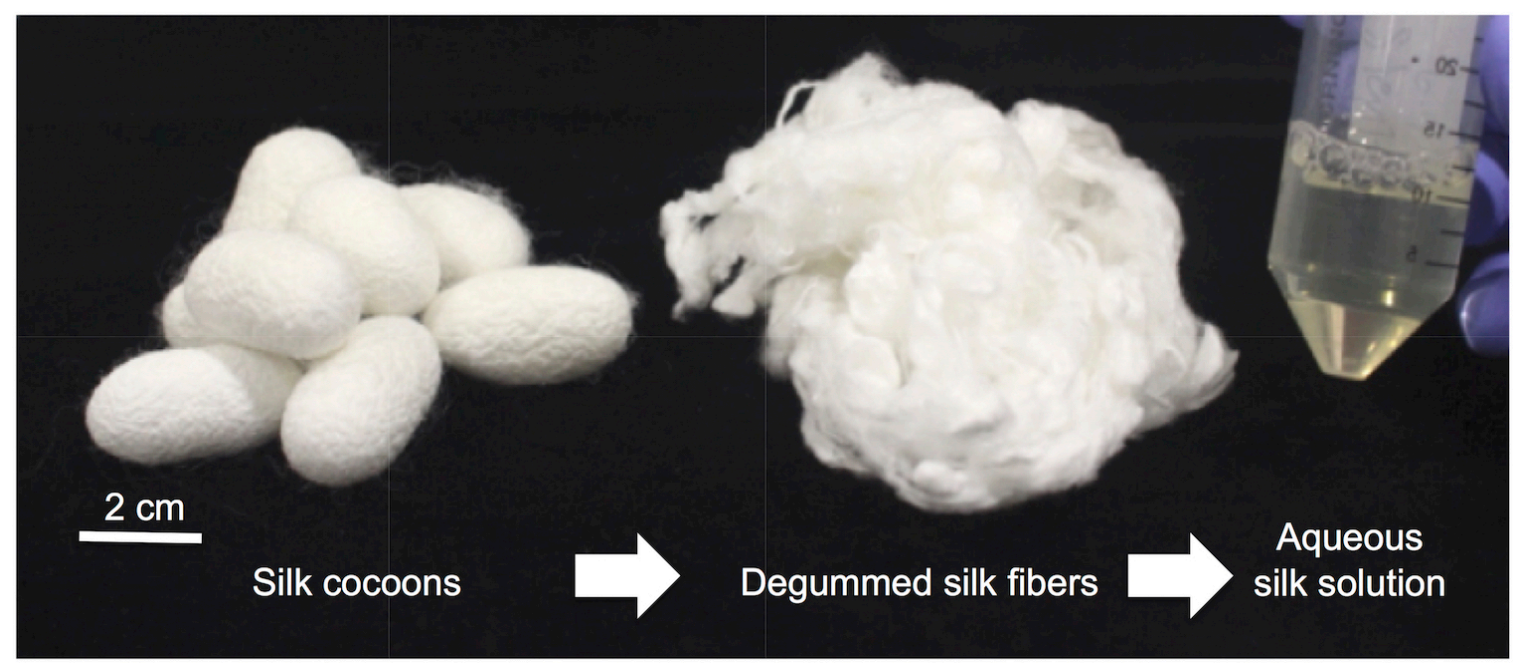

Figure 2. Reverse engineering of silk cocoons. Silk cocoons, degummed silk fibres and fully reverse engineered liquid silk. 
A

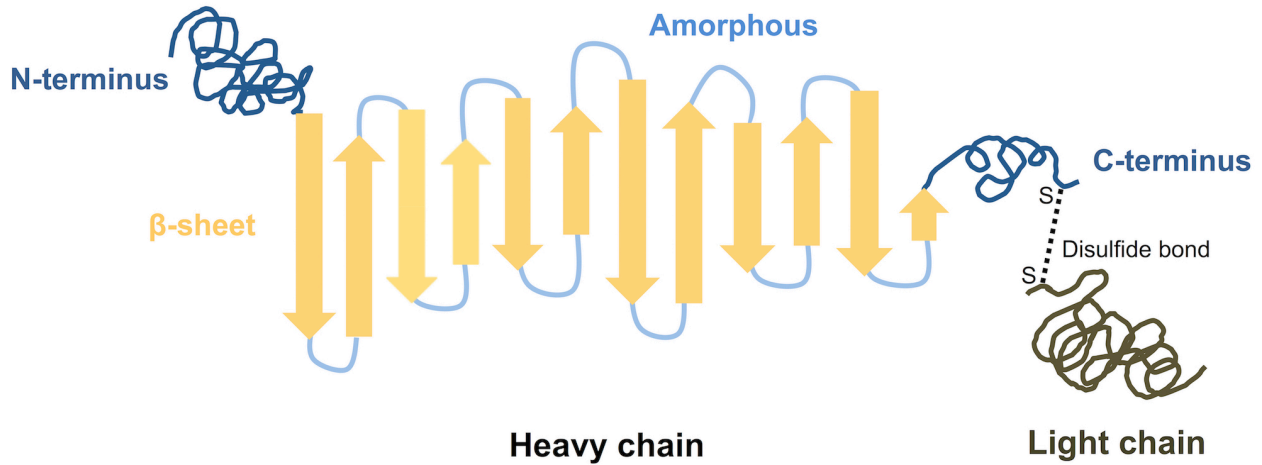

B

Cleavage site for chymotrypsin $\quad$ Cleavage site for papain

N-terminus

$\underset{\text { MRVKTFVILCCALQYVAYTNANINDFDEDYFGSDVTVQSSNTTDEIRDA }}{\mathbb{P} \nabla}$ SGAVIEEQITTKKMQRKNKNHGILGKNEKMIKTFVITTDSDGNESIVEED $\nabla_{\text {VLMKTLSDGTVAQSYVAADAGAYSQSGPYVSNSGYSTHQGYTSDFST }}^{\nabla} \nabla_{\nabla}^{\nabla}$ SAAV

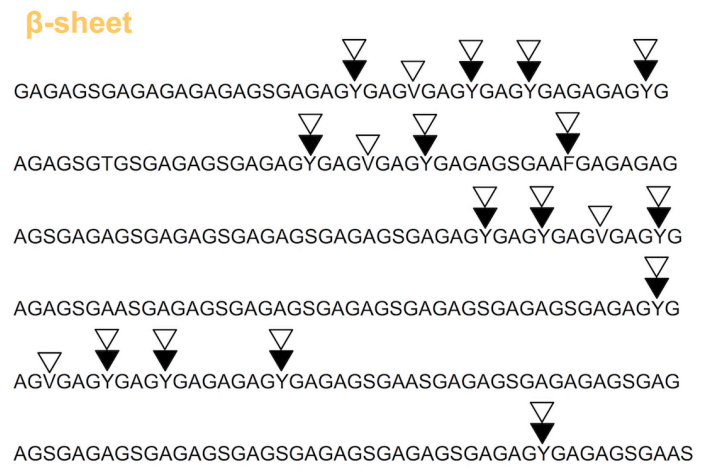

C

\begin{tabular}{lccccccc} 
& & \multicolumn{6}{c}{ Number of cleavage sites } \\
\cline { 3 - 8 } Enzymes & Cleavage sites & Heavy chain & N-terminus & -sheet & Amorphous & C-terminus & Light chain \\
Protease XIV & Typ, Phe,Trp, His, Lys, Arg & 348 & 27 & 222 & 84 & 15 & 41 \\
Chymotrypsin & Typ, Phe,Trp, Val, Ile, Leu & 434 & 41 & 291 & 88 & 14 & 81 \\
Papain & Lys, Arg & 26 & 12 & 0 & 4 & 10 & 15
\end{tabular}

Figure 3. The silk structure and cleavage site by proteolytic enzymes (reprinted with permission from [9]).
Amorphous

$\underset{\text { GAGAGAGAGTGSSGFGPYVANGGYSRREGYEYAWSSKSDFETGS }}{\nabla \nabla} \frac{\nabla}{\nabla \nabla \nabla \mathbb{8} \nabla}$

C-terminus

$\nabla$ VSYGAGRGYGQGAGSAASSVSSASSRSYDYSRRNVRKNCGIPRRQL
$\nabla \nabla$

$\underset{\text { VVKFRALPCVNC }}{\nabla}$

Light chain

$\underset{\text { MKPIFLVLLVATSAYAAPSVTINQYSDNEIPRDIDDGKASSVISRAWDYV }}{\mathbb{\nabla} \nabla} \underset{\nabla}{\nabla} \nabla \underset{\nabla}{\nabla}$

$\underset{\text { DDTDKSIALNVQEILKDMASQGDYASQASAVAQTAGIIAHLSAGIPGDA }}{\nabla} \nabla \underset{\nabla}{\nabla}$

$\underset{\nabla}{\nabla} \underset{\nabla}{\nabla} \underset{\nabla}{\nabla} \underset{\nabla}{\nabla} \nabla \underset{\nabla}{\nabla} \underset{\nabla}{\nabla}$

$\overbrace{\text { QLRYSVGPALGCAGGGRIYDFEAAWDALASSDSSFLNEEYCIVKRLYN }}^{\mathbb{Q}} \nabla \nabla$ SRNSQSNNIAAYITAHLLPPVAQVFHQSAGSITDLLRGVGNGNDATGLV ANAQRYIAQAASQVHV 

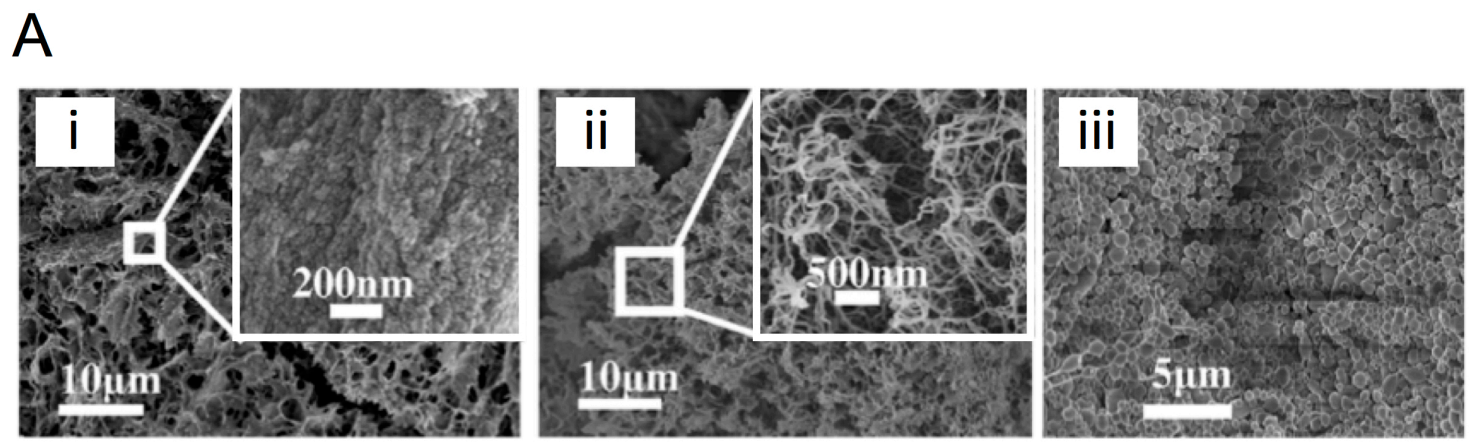

Figure 4. The structural characteristics of silk hydrogels. Scanning electron micrographs of different hydrogel morphologies derived from silk solution (i) of low and (ii) high concentration and (iii) silk e-gel. Note that the low and high silk concentration hydrogels (i.e. images $\mathrm{i}$ and ii) were allowed to self-assemble over several days whereas e-gels are formed within minutes. Reprinted with permission from [70]. Copyright American Chemical Society. 
A

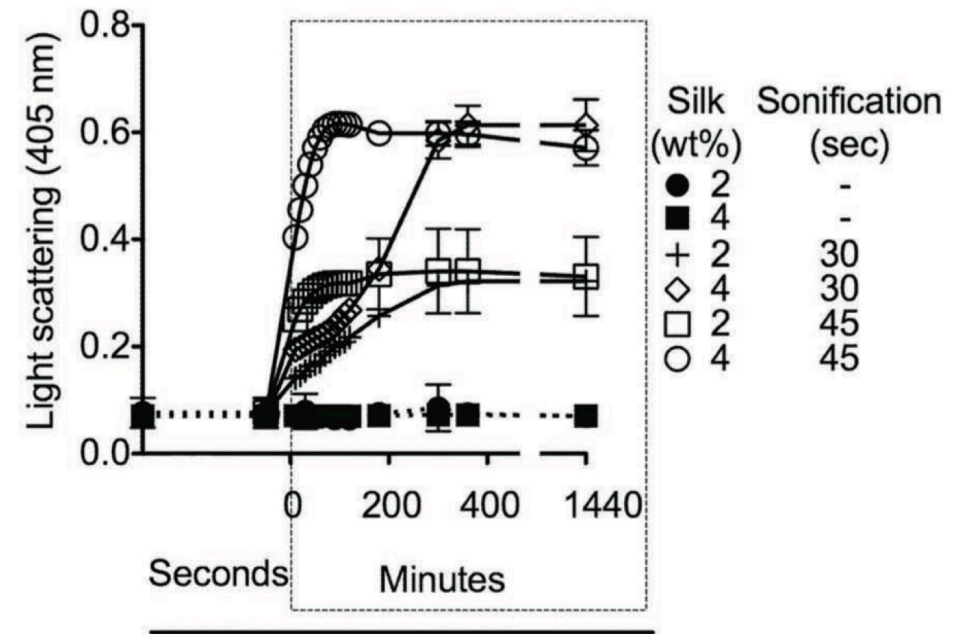

Time

c

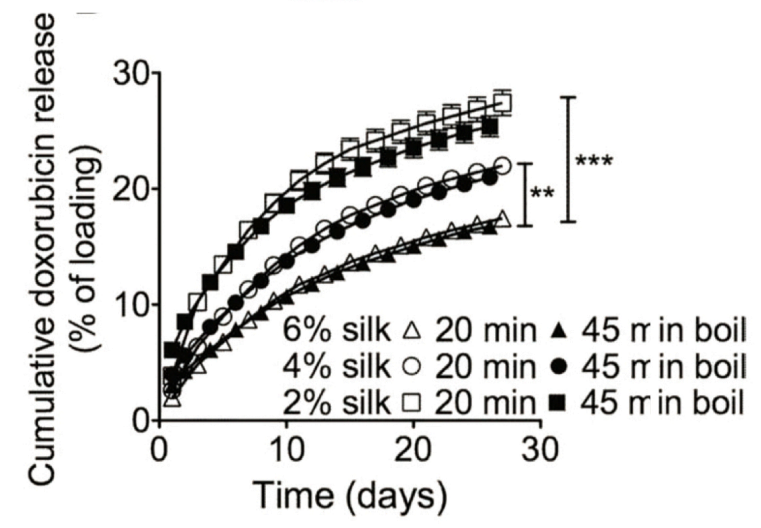

E $\quad \nabla$ Control

0 Doxorubicin i.v.

$\square$ Silk hydrogel + doxorubicin
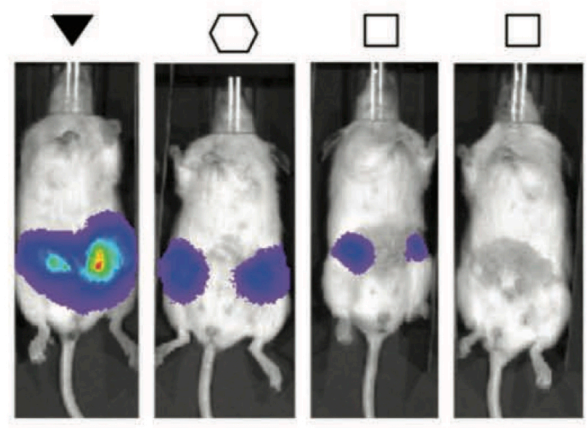

0.8

$\begin{array}{lll}0.6 & 0.4 & 0.2\end{array}$

Flux $\left(\mathrm{p} / \mathrm{sec} / \mathrm{cm}^{2} / \mathrm{sr}\right) \times 10^{9}$
B

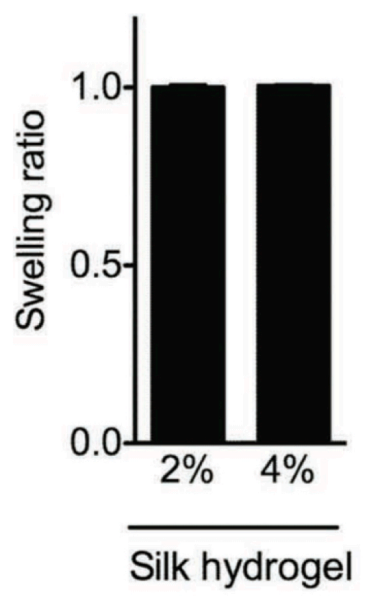

D
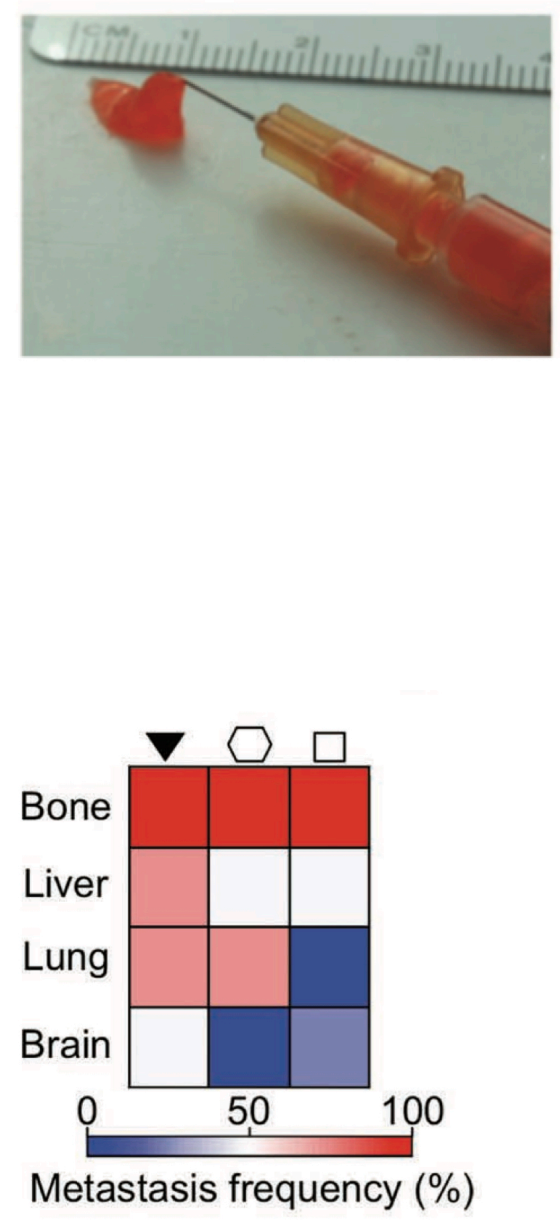
Figure 5. Self-assembling silk hydrogels for focal breast cancer focal therapy. (A) Impact of processing parameters on the solution-gel kinetics of silk (the open plot symbols 20 minute degummed silk, closed plot symbols 45 minutes degummed silk). (B) Silk hydrogels show no swelling during the solution-gel transition. (C) Doxorubicin release from different silk hydrogels. (D) Injectability of drug loaded silk hydrogels. (E) Response of MDA-MB-231 human breast cancer xenografts to treatment. Reproduced with permission from [39].
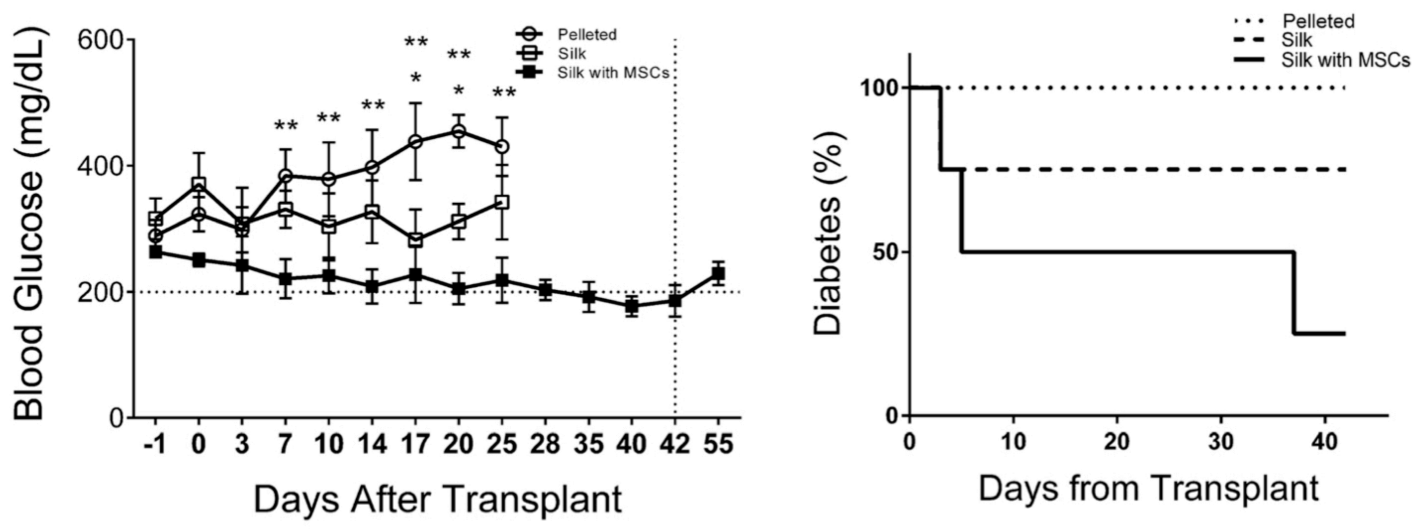

Figure 6. Performance of minimal pancreatic islets transplants in diabetic mice. (A) The treatment groups were (i) pelleted pancreatic islets (open circles), (ii) pancreatic islets delivered with a silk hydrogel (open squares) and (iii) pancreatic islets co-transplanted with mesenchymal stem cells and delivered with a silk hydrogel (closed squares). Only treatment group (iii) was able to control glucose levels and eliminated diabetes in $80 \%$ of mice (solid line). Reproduced with permission from [47]. 
